\title{
The Effect of Pregnancy and Lactation on Prolactinoma
}

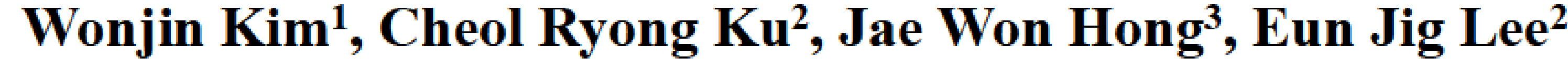

Department of Internal Medicine, Cha University College of Medicine, Seoul, Korea ${ }^{1}$, Department of Internal Medicine, Yonsei University College of Medicine, Seoul, Korea ${ }^{2}$, Department of Internal Medicine, Ilsan-Paik Hospital, College of Medicine, Inje University, Koyang, Gyeonggi-do, Korea ${ }^{2}$

\section{Introduction \& objective}

Prolactinoma is the most common pituitary adenoma which accounts for $45 \%$ of all pituitary tumors.

Hyperprolactinemia in these patients is associated with anovulation and infertility.

$>$ Treatment of hyperprolactinemia with dopamine agonists, such as bromocriptine and cabergoline, is an important mode

of treatment which can restore ovulation in about $90 \%$ of cases.

$>$ In normal pregnancy

- Pituitary gland increases in their size up to $\underline{136 \%}$ throughout this period.

- Serum prolactin level is also rises at the end of pregnancy

In patients with prolactinoma

- About 10 to $35 \%$ of patients showed remission of hyperprolactinemia.

- Recently, about $68 \%$ of subjects showed normalization of serum prolactin level and no tumor growth up to 60 months after pregnancy.

Bromocriptine

$>$ Used to be the first choice of treatment in prolactinoma

$>$ More than 6000 pregnancies were reported

$>11 \%$ of spontaneous abortions

$>3.5 \%$ of neonatal malformation

Cabergolin

$>$ Treatment of choice for prolactinoma

$>$ Longer half-life \& higher effectiveness

$>$ Fetal exposure to cabergoline at conception is not affected to miscarriage or fetal malformation

$>$ Breastfeeding did not increase the recurrence of hyperprolactinemia.

$>$ In this study, we aimed to analyze

$>$ the safety of exposure to dopamine agonists in the early period of pregnancy

$>$ the effect of lactation in the aspects of adenoma size and serum prolactin levels in Korean women with prolactinoma.

\section{Materials and Methods}

$>$ Study population

$>$ Female patients over 18 years old

P Previously diagnosed prolactinoma and treated with bromocriptine or cabergoline

$>$ Medical record review from January, 2005 to March, 2013

> Pregnancy type, age at conception, previous and current medical history and treatment duration, postpartum

medication, tumor size and extension by MRI, and serum prolactin level

$>$ Sella dynamic MRI and serum prolactin level

$>$ Initial visit

Three-month follow-up after the intitition of dopamine agonists

> Within 1 year before preanney

Within 3 months after childbirth

Within 6 months ffer lactation

\section{$\underline{\text { Results }}$}

Table 1. Baseline characteristics of study population

\begin{tabular}{lc}
\hline & Profile \\
\hline Patients (n) & 50 \\
Patients' age (years, $\mathrm{n}=50)$ & $34.2 \pm 3.8$ \\
Pregnancy counts & 65 \\
Age at conception (years) & $31.7 \pm 3.4$ \\
\hline Lactation [n (\%)] & $39(70.9)$ \\
Duration of lactation (months) & $4.8 \pm 4.4$ \\
\hline Drug dosage & \\
$\quad$ Bromocriptine (mg/day, $\mathrm{n}=33)$ & $7.43 \pm 4.4$ \\
$\quad$ Cabergoline (mg/week, $\mathrm{n}=22)$ & $1.49 \pm 0.7$ \\
\hline Initial adenoma size $(\mathrm{cm})$ & $0.99 \pm 0.5$ \\
Initial prolactin level $(\mathrm{ng} / \mathrm{mL})$ & $177.5 \pm 522.4$ \\
Microadenoma [n $(\%)]$ & $34(61.8)$ \\
Macroadenoma $[\mathrm{n}(\%)]$ & $21(38.2)$ \\
\hline
\end{tabular}

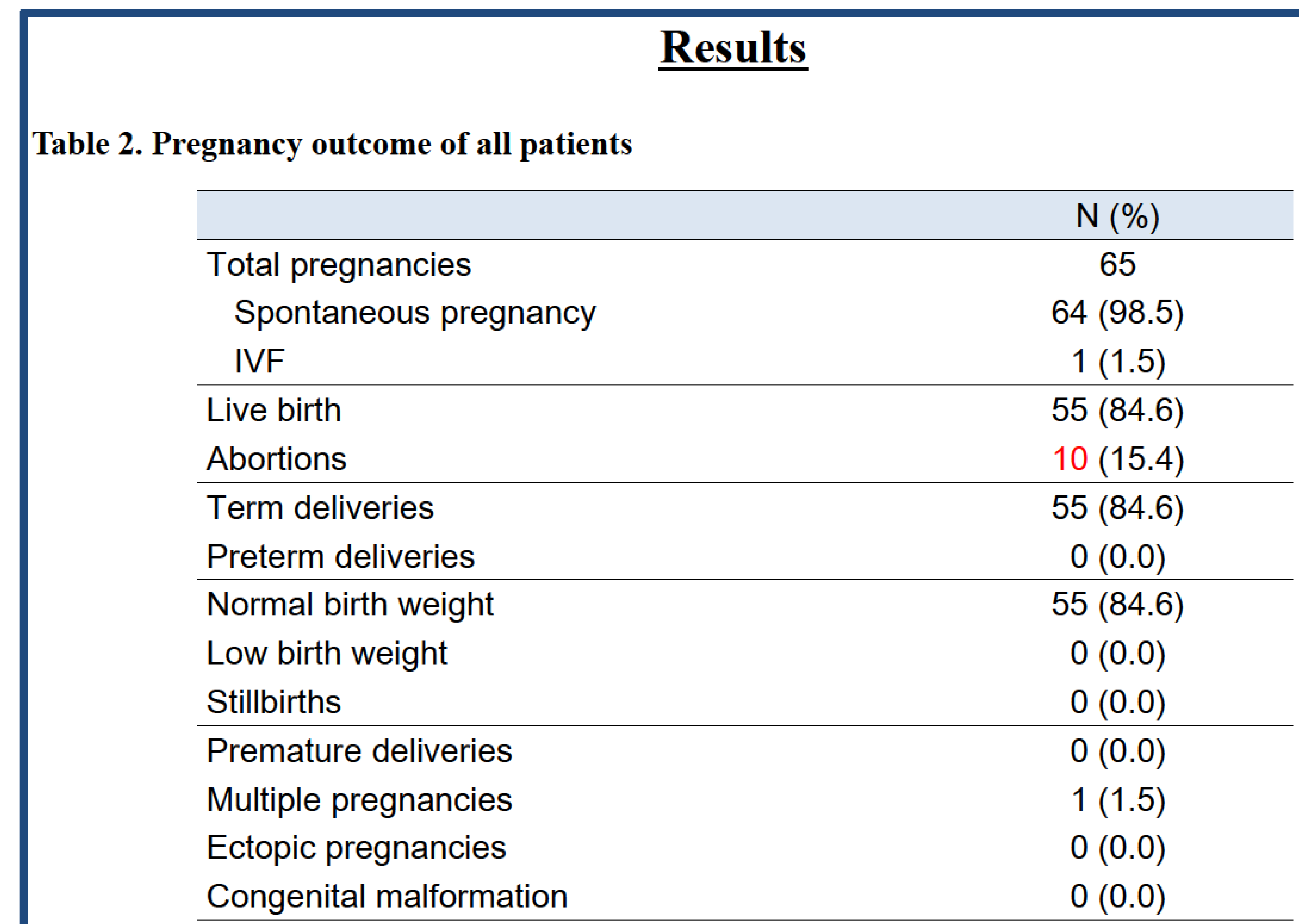

Figure 1. Changes of adenoma size and prolactin level
Adenoma size

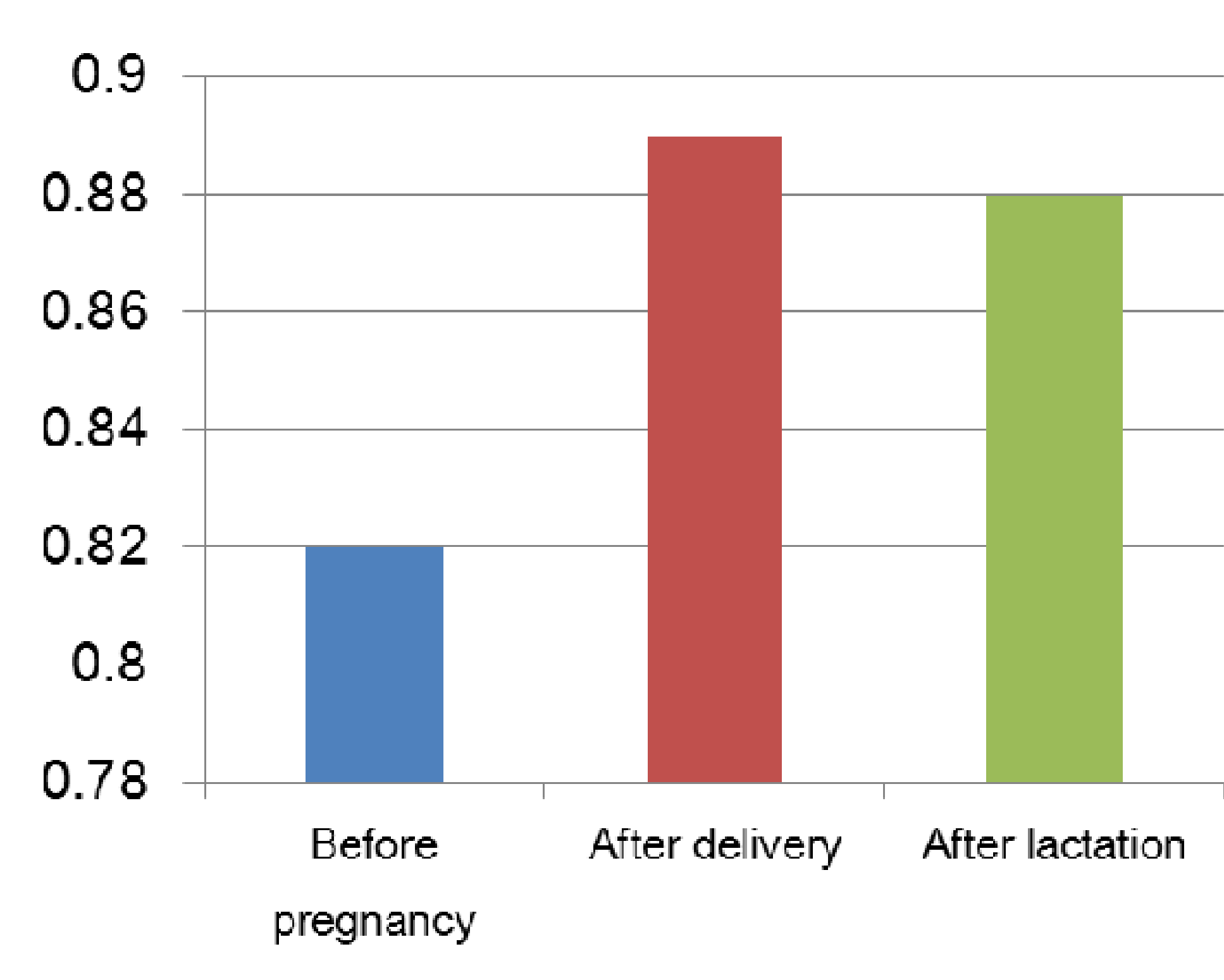

Serum prolactin level

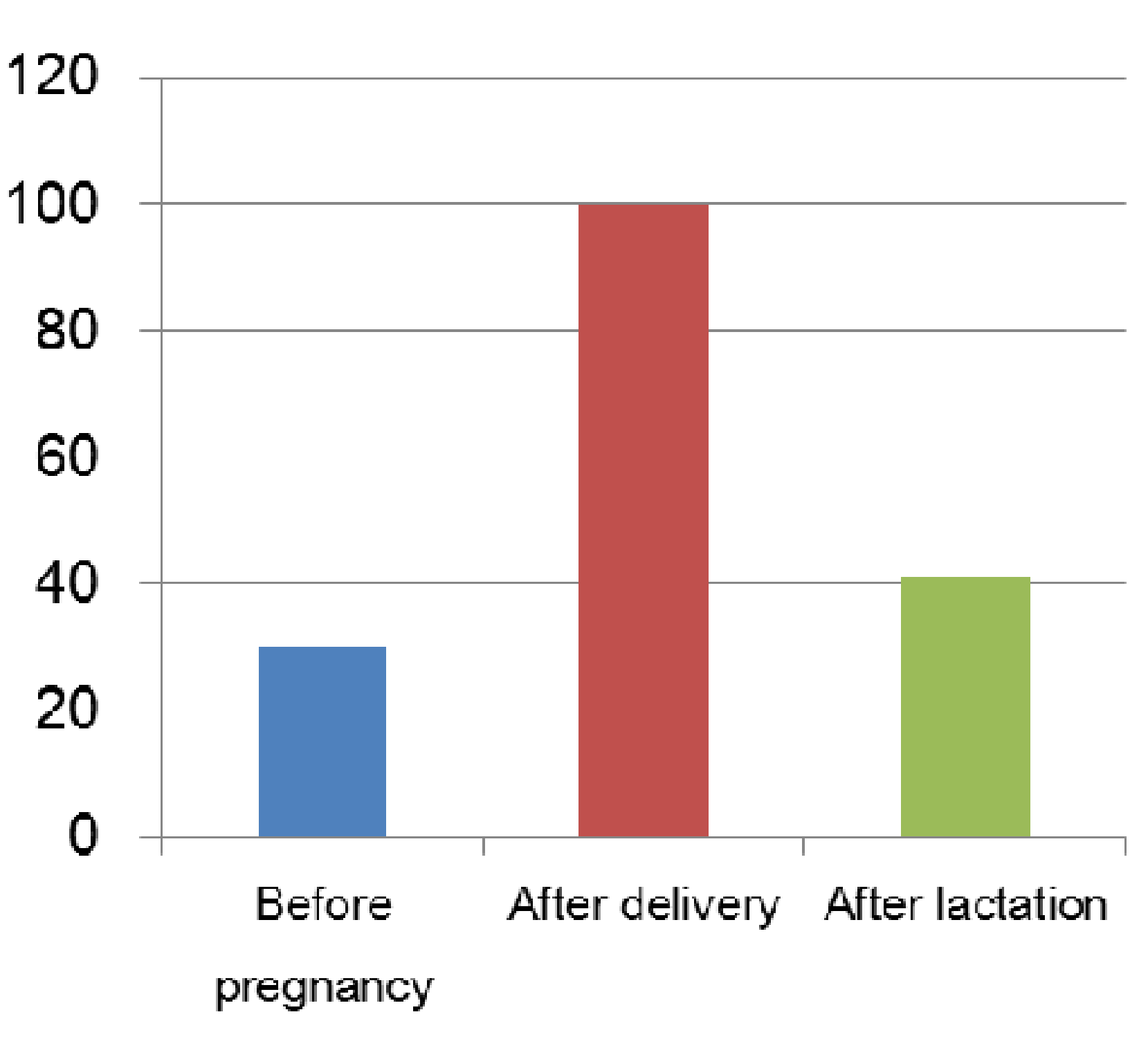

Table 3. Comparisons of several parameters in patients whose adenoma size decreased and/or no change or increased.

\begin{tabular}{lccc}
\hline & $\begin{array}{c}\text { Pts. With increased } \\
\text { adenoma size } \\
(\mathrm{n}=2)\end{array}$ & $\begin{array}{c}\text { Pts. with decreased or no } \\
\text { change of adenoma size } \\
(\mathrm{n}=14)\end{array}$ & P-value \\
\hline Age & $36.0 \pm 0.0$ & $34.8 \pm 4.0$ & 0.333 \\
Age at conception & $31.5 \pm 0.7$ & $32.1 \pm 3.9$ & 1.000 \\
No. of pregnancy & $1.0 \pm 0.0$ & $1.4 \pm 0.6$ & 0.500 \\
No. of delivery & $1.0 \pm 0.0$ & $1.1 \pm 0.4$ & 0.817 \\
Duration of lactation (month) & $7.5 \pm 5.0$ & $4.3 \pm 4.5$ & 0.333 \\
Drug duration before pregnancy & $7.5 \pm 0.7$ & $22.5 \pm 19.0$ & 0.600 \\
Drug cessation time after pregnancy & 4.0 & $6.0 \pm 1.5$ & 0.308 \\
confirmation (pregnancy week) & $1(50.0 \%)$ & $7(50.0 \%)$ & \\
Microadenoma (\%) & $1.23 \pm 0.4$ & $1.17 \pm 0.5$ & 0.700 \\
Initial adenoma size(cm) & $0.90 \pm 0.1$ & $1.04 \pm 0.5$ & 0.933 \\
Adenoma size before pregnancy $(\mathrm{cm})$ & $1.23 \pm 0.4$ & $1.09 \pm 0.4$ & 0.600 \\
Adenoma size after delivery $(\mathrm{cm})$ & $1.19 \pm 0.4$ & $0.84 \pm 0.5$ & 0.200 \\
Adenoma size after lactation $(\mathrm{cm})$ & $178.4 \pm 19.9$ & $364.7 \pm 1001.4$ & 0.200 \\
Initial serum prolactin level $(\mathrm{ng} / \mathrm{mL})$ & $25.8 \pm 33.7$ & $41.3 \pm 50.9$ & 0.817 \\
Serum prolactin level before pregnancy & $128.3 \pm 112.1$ & $129.9 \pm 70.1$ & 0.933 \\
(ng/mL) & $40.8 \pm 50.0$ & $41.9 \pm 43.8$ & 0.700 \\
Serum prolactin level after delivery $(\mathrm{ng} / \mathrm{mL})$ & & & \\
Serum prolactin level after lactation $(\mathrm{ng} / \mathrm{mL})$ & & &
\end{tabular}

\section{Conclusion}

$>$ This study revealed the effect of early exposure of dopamine agonists did not affect to miscarriage or neonatal malformations.

$>$ In addition, breastfeeding is not associated with the recurrence of hyperprolactinemia and enlargement of pituitary tumor

$>$ More patients with multi-center, prospective, long-term study will be needed to confirm the safety of dopamine agonists in prolactinoma patients on pregnancy and lactation. 